\title{
Outcomes and risk factors associated with endophthalmitis after intravitreal injection of anti-vascular endothelial growth factor agents.
}

\author{
Chirag P Shah \\ Ophthalmic Consultants of Boston, Boston, MA, United States \\ Sunir J Garg \\ Retina Service of Wills Eye Institute, Thomas Jefferson University \\ James F Vander \\ Retina Service of Wills Eye Institute, Thomas Jefferson University \\ Gary C Brown

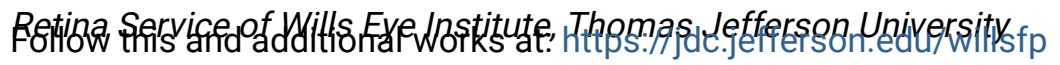 \\ ipard of thais seththalmology Commons

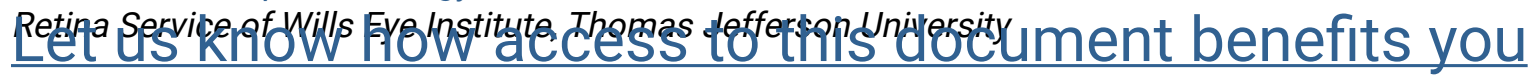

\section{Ree}

Shah, Chirag P; Garg, Sunir J; Vander, James F; Brown, Gary C; Kaiser, Richard S; Haller, Julia A; and The Post-Injection Endophthalmitis (PIE) Study Team, "Outcomes and risk factors associated with endophthalmitis after intravitreal injection of anti-vascular endothelial growth factor agents." (2011). Wills Eye Hospital Papers. Paper 10.

https://jdc.jefferson.edu/willsfp/10

This Article is brought to you for free and open access by the Jefferson Digital Commons. The Jefferson Digital Commons is a service of Thomas Jefferson University's Center for Teaching and Learning (CTL). The Commons is a showcase for Jefferson books and journals, peer-reviewed scholarly publications, unique historical collections from the University archives, and teaching tools. The Jefferson Digital Commons allows researchers and interested readers anywhere in the world to learn about and keep up to date with Jefferson scholarship. This article has been accepted for inclusion in Wills Eye Hospital Papers by an authorized administrator of the Jefferson Digital Commons. For more information, please contact: JeffersonDigitalCommons@jefferson.edu. 


\section{Authors}

Chirag P Shah, Sunir J Garg, James F Vander, Gary C Brown, Richard S Kaiser, Julia A Haller, and The PostInjection Endophthalmitis (PIE) Study Team 
As submitted to:

Ophthalmology

And later published as:

Outcomes and Risk Factors Associated with Endophthalmitis after Intravitreal Injection of Anti-Vascular Endothelial Growth Factor Agents

\section{Volume 118, Issue 10, October 2011, Pages 2028-2034 DOI: 10.1016/j.ophtha.2011.02.034}

Chirag P. Shah, MD, MPH ${ }^{1,2}$, Sunir J. Garg, MD², James F. Vander, MD², Gary C. Brown, MD, MBA ${ }^{2}$, Richard S. Kaiser, $\mathrm{MD}^{2}$, Julia A. Haller, $\mathrm{MD}^{2}$, for The PostInjection Endophthalmitis (PIE) Study Team*

${ }^{1}$ Ophthalmic Consultants of Boston, Boston, MA

${ }^{2}$ Wills Eye Institute, Retina Service, Philadelphia, PA

*Darrell E. Baskin, $\mathrm{MD}^{2}$, Jeremy D. Wolfe, $\mathrm{MD}^{2}$, Paul Baker, $\mathrm{MD}^{2}$, Allen Chiang, $\mathrm{MD}^{2}$, Eugene Milder, $\mathrm{MD}^{2}$, William Benson, $\mathrm{MD}^{2}$, Jay Federman, $\mathrm{MD}^{2}$, David Fischer, $\mathrm{MD}^{2}$, Allen C. Ho, $\mathrm{MD}^{2}$, Jason Hsu, $\mathrm{MD}^{2}$, Alfred Lucier, $\mathrm{MD}^{2}$, Joseph I. Maguire, $\mathrm{MD}^{2}$, J. Arch McNamara, $\mathrm{MD}^{2}$, Carl D. Regillo, $\mathrm{MD}^{2}$, Lov Sarin, $\mathrm{MD}^{2}$, Arunan Sivalingam, $\mathrm{MD}^{2}$

Proprietary interests: none

Running head: Post-injection Endophthalmitis

Correspondence to: Sunir J. Garg, MD

sunirgarg@yahoo.com

Financial support: none

Online only materials: This article contains online-only material. The following should appear online-only: Table 1

Video: no 
Shah, et al. Post-injection Endophthalmitis. Pg. 2 of 14

1

2 Key Words: endophthalmitis, anti-VEGF injection, bevacizumab, ranibizumab, AMD, 3 risk factor

revised 12/2/11 
Objective: Describe outcomes of and risk factors for endophthalmitis following intravitreal anti-VEGF injection.

Design: Single-center, consecutive, case series and retrospective case-control study

Participants: Between 1/1/09 and 5/31/10, 16 vitreoretinal surgeons administered a total of 27,736 injections. During this period, twenty-three cases of presumed infectious endophthalmitis occurred. Each surgeon used their own preferred injection technique.

\section{Intervention:}

Vitreous and/or aqueous tap with intravitreal antibiotic injection and subsequent topical antibiotic and steroid drops.

Main Outcome Measures: Visual acuity, bladed lid speculum use, conjunctival displacement, hemisphere of injection, bevacizumab vs. ranibizumab, and infectious organism.

Results: Seven of 23 cases were culture-positive; three grew coagulase negative Staphylococcus. All cases presented with pain and vitritis on average 3.4 days (range $1-$ 6) after injection, with no difference between culture-positive and culture-negative groups. Eighteen of 23 cases ( $78 \%$ ) had a hypopyon. 16 of 23 cases returned to baseline vision (+/- 2 lines) within three months. Neither lid speculum use $(0.10 \%$ vs. $0.066 \%$ in the no use group, $\mathrm{p}=0.27)$, conjunctival displacement $(0.11 \%$ vs. $0.076 \%$ no displacement, $\mathrm{p}=0.43)$, hemisphere of injection $(0.11 \%$ superior vs. $0.079 \%$ inferior, $\mathrm{p}=$ $0.56)$, or bevacizumab vs. ranibizumab $(0.11 \%$ vs $0.066 \%, \mathrm{p}=0.21)$ affected risk.

Analysis of only culture positive results yielded similar results. There was no statistically significant difference between the proportion of culture-negative cases after bevacizumab $(83 \%)$ versus ranibizumab injection $(55 \%, \mathrm{p}=0.13)$.

Conclusion: Most patients who develop presumed infectious endophthalmitis after antiVEGF injection regained baseline vision after treatment. Bladed lid speculum use, conjunctival displacement, hemisphere of injection, and type of anti-VEGF agent did not affect risk. We did not detect a difference in culture-negative endophthalmitis rates after bevacizumab versus ranibizumab injection. Neither the presence of pain, vitritis, decreased vision, or hypopyon, nor the interval between injection and development of symptoms, differentiated culture-positive from culture-negative cases. As a subgroup of patients have poor outcomes, a low threshold for vitreous tap with intravitreal antibiotic injection may be warranted. 
Introduction:

Intravitreal anti-vascular endothelial growth factor (anti-VEGF) agents have revolutionized the treatment of neovascular age-related macular degeneration (AMD). The use of these medications continues to increase as their indications expand, including for diseases such as retinal vein occlusions ${ }^{1,2}$, neovascular glaucoma ${ }^{3}$, and diabetic macular edema ${ }^{4}$.

Infectious endophthalmitis remains one of the most feared complications of intravitreal injections. Endophthalmitis can lead to apoptosis of ganglion cells, bipolar cells, and photoreceptors ${ }^{5}$, or to retinal detachment, which can all lead to significant vision loss or to loss of the eye.

Few clinical studies describe visual outcomes after post-injection endophthalmitis $^{6-8}$ or identify modifiable risk factors to prevent infection. Further, there is debate regarding the clinical distinction between infectious and non-infectious endophthalmitis, with some authors positing that absence of pain supports a noninfectious etiology 9,10 . This study evaluates a large series of endophthalmitis cases developing after anti-VEGF injection and assesses outcomes and risk factors.

\section{Patients and Methods}

Overview:

Institutional Review Board approval was obtained from Wills Eye Institute. During an infection surveillance program, the authors prospectively recorded cases of endophthalmitis occurring after intravitreal injection of bevacizumab or ranibizumab between January 1, 2009 and May 31, 2010. Charts from these cases were retrospectively reviewed at the conclusion of the surveillance period. All injections were performed at a single, retina-only practice by 16 different vitreoretinal specialists with 16 different offices. The total number of intravitreal bevacizumab and ranibizumab injections was determined using billing data, allowing a retrospective case-control analysis for risk factors.

\section{Injection technique:}

All eyes were prepped in a standardized fashion. Briefly, eyes were anesthetized with topical drops (e.g., proparacaine 0.5\% [Ophthetic, Allergan, Inc.]), a topical antibiotic (e.g., ofloxacin $0.3 \%$ [Ocuflox, Allergan, Inc.]), topical 5\% povidone-iodine (Betadyne, Alcon Labs), viscous anesthetic (e.g., tetracaine solution 0.5\% [TetraVisc, OCuSoft, Inc.]), and another drop of topical 5\% povidone-iodine prior to injection. Rarely, subconjunctival lidocaine $2 \%$ was substituted for viscous anesthesia. The eyelashes were not prepped and a sterile drape was not used. Pre-injection antibiotics were not used.

Each vitreoretinal specialist administered anti-VEGF injections through the pars plana, $3.5-4.0 \mathrm{~mm}$ from the limbus with a 30- or 31-gauge needle using his or her preferred technique. Physicians were asked to consistently use his or her preferred injection technique for the duration of the infection surveillance period, and periodic monitoring was performed to ascertain whether there was identifiable change in technique. Variables included bladed lid speculum use, conjunctival displacement with a sterile cotton tip applicator prior to injection, and superior versus inferior hemisphere of injection. Physicians not using a lid speculum employed variable techniques to expose 
the globe, including gloved or ungloved fingers to open the lids, an assistant's gloved or ungloved fingers, or simply instructed patients to open their eyelids widely. Those not displacing conjunctiva with a cotton tip applicator injected straight through conjunctiva and sclera into the vitreous. Patients were prescribed a topical antibiotic to use four times a day for four days post-injection. The specific antibiotic was per the preference of the injecting physician.

\section{Tap and inject protocol:}

All eyes that developed presumed infectious endophthalmitis were sent to Wills Eye Institute for immediate tap of the vitreous through the pars plana with injection of intravitreal antibiotics (tap and inject). No patients were treated at satellite offices. The vitreous tap consisted of insertion of a 25-gauge needle into the vitreous cavity with attempted aspiration of vitreous in all patients. If adequate vitreous fluid was unable to be obtained, an aqueous tap was performed. All samples were sent to the department of microbiology at Thomas Jefferson University Hospital, Philadelphia, PA, for gram stain, cultures, and sensitivities. Patients then received intravitreal vancomycin $(1 \mathrm{mg} / 0.1 \mathrm{~mL})$ and intravitreal ceftazidime $(2 \mathrm{mg} / 0.1 \mathrm{~mL})$. Penicillin allergic patients received intravitreal amikacin $(400 \mathrm{mcg} / 0.1 \mathrm{~mL})$ instead of intravitreal ceftazidime. All patients were then placed on fortified vancomycin $(25 \mathrm{mg} / \mathrm{mL})$, fortified tobramycin $(15 \mathrm{mg} / \mathrm{mL})$, and prednisolone acetate $1 \%$ drops every hour, as well as atropine sulfate $1 \%$ drops twice a day. Patients were followed daily until they had evidence of clinical improvement, at which time the drops were slowly tapered and examination intervals were gradually extended. Antibiotic drops also were modified based on culture sensitivity data.

\section{Inclusion and exclusion criteria:}

All eyes with presumed infectious endophthalmitis warranting tap and inject were included in this case series. The criteria for tap and inject were dependent on the judgment of individual vitreoretinal specialists, but universally included decreased visual acuity, the presence of pain, and the presence of vitritis within one week of intravitreal anti-VEGF injection. Patients not included in this case series were those with mild postinjection anterior chamber inflammation (1+ or less), who improved on topical corticosteroid and antibiotic drops without undergoing tap and inject.

\section{Endophthalmitis surveillance log:}

One researcher (CPS) recorded data for all patients undergoing tap and inject in an infection surveillance log. These data included the presence of pain, vitritis, and/or hypopyon, visual acuity before the causative injection and at time of tap and inject (Snellen acuity, not best corrected), date of causative anti-VEGF injection, date of tap and inject, office location, injecting vitreoretinal surgeon, type of anti-VEGF injection (bevacizumab versus ranibizumab), lot number, underlying retinal diagnosis, number of prior anti-VEGF injections, lens status, source of tap (vitreous or aqueous), identified organism, and antibiotic specificities. At the end of the surveillance period, charts were retrospectively reviewed to collect follow-up data.

\section{Analysis of case series and case-control study:}


Clinical variables of presumed infectious endophthalmitis were analyzed using Excel (Microsoft, Redmond, WA). These features included the presence of pain, hypopyon, vitritis, decreased vision, and duration between causative anti-VEGF injection and tap and inject. Outcome data included return of baseline visual acuity (plus or minus two lines of Snellen acuity, not best-corrected) and need for pars plana vitrectomy.

To evaluate risk factors for developing endophthalmitis, the authors conducted a retrospective case-control analysis. The total number of bevacizumab and ranibizumab injections administered was determined using billing data. The number of anti-VEGF injections was also stratified by office location and injecting vitreoretinal surgeon.

Several risk factors for presumed infectious endophthalmitis after anti-VEGF injection were examined. These included bladed lid speculum use, conjunctival displacement with a sterile cotton tip applicator prior to injection, superior versus inferior hemisphere of injection, the use of bevicizumab versus ranibizumab, office location, injecting vitreoretinal specialist, and lot number of the specific anti-VEGF agent. A two-sample test of proportion was performed using Stata 9 (College Park, TX). Analysis was done for all cases of presumed infectious endophthamitis and further stratified for culturepositive and culture-negative cases.

\section{$\underline{\text { Results }}$}

\section{Clinical Features}

During the 17-month study period, a total of 27,736 consecutive intravitreal antiVEGF injections were administered, including 10,958 bevacizumab and 16,778 ranibizumab injections. Twenty-three of these cases underwent emergent tap and inject for presumed infectious endophthalmitis $(0.083 \%, 95 \%$ confidence interval $0.049 \%$ to $0.12 \%$ ). Twenty-one of these eyes received anti-VEGF injection for neovascular AMD, while two were treated for macular edema secondary to branch retinal vein occlusion.

All cases of presumed infectious endophthalmitis presented with pain, vitritis, and decreased visual acuity. Most cases had a hypopyon at time of tap and inject (18 of 23 eyes, $78 \%)$. Five of seven culture-positive cases presented with hypopyon $(71 \%$, $\mathrm{p}=\mathrm{XXX})$.

There was an average of 3.4 days (range 1 to 6 days) between administration of anti-VEGF injection and emergent tap and inject. This average was similar between culture-negative ( 3.5 days, range 1 to 6 days) and culture-positive cases (3.1 days, range 1 to 5 days, $\mathrm{p}=0.54$ ). One culture-negative case presenting 17 days after injection was excluded from this analysis because the patient's nursing home delayed seeking medical attention.

Vitreous tap was performed in all cases, and an adequate specimen was obtained in 14 of 23 cases. When the vitreous tap was unsuccessful, an aqueous tap was performed successfully in the remaining 9 of 23 cases. An infectious organism was identified from vitreous and/or aqueous biopsy in $30.4 \%$ of patients ( 7 of 23 ), for a culture-positive endophthalmitis rate of $0.025 \%$ per injection. Causative organisms included three cases of coagulase negative staphylococci, and one case of each Staphylococcus aureus, Streptococcus viridans, Streptococcus mitus, and Enterococcus faecalis.

\section{Visual Outcomes}


Most cases (16 of 23, 70\%) returned to baseline vision (+/- 2 lines) within three months (see Table 1, available at http://aaojournal.org). Four more cases returned to baseline vision at six months; a total of $83 \%$ of cases had recovery of baseline vision. Specifically, the three eyes that did not return to baseline were as follows: the vision of one patient dropped from 20/300 to no light perception after retinal detachment with subsequent retinal detachment repair, one from 20/40 to counting fingers after retinal detachment repair, one from 20/400 to counting fingers, and one from 20/50 to 20/100. Four of 23 cases (17\%) underwent pars plana vitrectomy three days to 3 weeks after initial tap and inject for retinal detachment, vitreous hemorrhage, or worsening endophthalmitis.

Of the seven culture-positive cases, four returned to baseline vision by three months and an additional case returned by six months (71\%). Of the two culture-positive eyes not returning to baseline vision, both underwent subsequent pars plana vitrectomy for retinal detachment. These eyes grew Streptococcus viridans and Streptococcus mitus, respectively.

Of the 16 culture-negative cases, 13 returned to baseline vision by three months with another two returning by six months $(94 \%)$. There was no significant difference in the visual recovery rate between culture positive and culture-negative cases $(\mathrm{p}=0.14)$. Of note, one patient developed pain, decreased vision, and hypopyon twice after sequential bevacizumab injection (patient's third and fourth injections). During the first episode, the patient underwent tap and inject three days after causative bevacizumab injection and improved to baseline visual acuity at six weeks. During the second episode, the patient was treated initially with hourly prednisolone acetate drops and had continued worsening of inflammation. The patient underwent tap and inject three days after causative bevacizumab injection, and did not regain baseline visual acuity at six months. This eye was counted twice, once for each episode.

\section{Risk Factors}

Cases of endophthalmitis occurred in nine of 16 offices by nine of 16 injecting vitreoretinal surgeons. There were no clusters of endophthalmitis with any individual treating physician or in any particular office location. There were no trends associated with lot numbers of bevacizumab or ranibizumab injections.

No modifiable risk factors were identified (see Table 2). Neither lid speculum use [0.10\% (13 of 12,500$)$ vs. $0.066 \%$ (10 of 15,236$)$ in the no use group, $p=0.27,95 \%$ confidence interval of the difference -0.031 to $0.11 \%]$, conjunctival displacement [0.11\% ( 6 of 5,421$)$ vs. $0.076 \%$ ( 17 of 22,315 ) no displacement, $\mathrm{p}=0.43,95 \%$ confidence interval of the difference -0.061 to $0.13 \%]$,], hemisphere of injection $[0.11 \%(4$ of 3,683$)$ superior vs. $0.079 \%$ (19 of 24,053) inferior, $\mathrm{p}=0.56,95 \%$ confidence interval of the difference -0.082 to $0.14 \%]$,], or bevacizumab $(0.11 \%, 12$ of 10,958$)$ vs. ranibizumab $(0.066 \%, 11$ of $16,778, \mathrm{p}=0.21,95 \%$ confidence interval of the difference -0.030 to $0.12 \%]$,) affected risk. Results were similar with analysis of only culture-positive cases $[0.032 \%$ ( 4 of 12,500$)$ vs. $0.020 \%$ ( 3 of 15,236$)$ in the no speculum group $(\mathrm{p}=0.52)$, $0.018 \%$ ( 1 of 5,421 ) vs. $0.027 \%$ ( 6 of 22,315 ) in the no conjunctival displacement group $(\mathrm{p}=0.73), 0.054 \%$ (2 of 3,683) superior vs. $0.021 \%$ (5 of 24,053) inferior hemisphere of injection ( $\mathrm{p}=0.23$ ), and $0.018 \%$ ( 2 of 10,958$)$ post-bevacizumab vs. $0.030 \%$ (5 of $16,778)$ post-ranibizumab $(\mathrm{p}=0.55)]$. The proportion of culture-negative cases was 
similar after bevacizumab $(83 \%, 10$ of 12$)$ and ranibizumab injection $(55 \%, 6$ of $11, \mathrm{p}=$ $0.13)$.

Power calculations revealed that 101,958 injections evenly split between two groups would be needed to detect a difference between $0.05 \%$ and $0.10 \%$ with an alpha of 0.05 and a beta of 0.20 .

\section{Discussion}

This large, single-center cases series and case-control study evaluated cases with presumed infectious endophthalmitis occurring after intravitreal anti-VEGF injection.

Overall, we detected 23 cases of endophthalmitis after 27,736 injections for an incidence of $0.083 \%$. All cases presented with pain, decreased visual acuity, and vitritis three to four days after intravitreal anti-VEGF injection; most eyes had hypopyon. These features did not help distinguish between culture-positive and culture-negative cases. Most cases returned to baseline visual acuity within three to six months, though some suffered significant visual loss. There were no modifiable risk factors for post-injection endophthalmitis, including the use of a bladed lid speculum, conjunctival displacement with a sterile cotton tip applicator, superior versus inferior hemisphere of injection, and the use of bevacizumab versus ranibizumab.

The reported rates of endophthalmitis after intravitreal anti-VEGF injection vary between institutions, study designs, and definitions of endophthalmitis. Our rate is consistent with other large prospective trials. The Minimally Classic/Occult Trial of the Anti-VEGF Antibody Ranibizumab in the Treatment of Neovascular AMD (MARINA) study reported an endophthalmitis incidence of $0.05 \%$ ( 5 cases per 10,443 injections) ${ }^{11}$, identical to the rate reported in the Anti-VEGF Antibody for the Treatment of Predominantly Classic Choroidal Neovascularization in AMD (ANCHOR) study ${ }^{12,13}$ (3 cases per 5,921 injections). However, 14 patients in the MARINA trial and 10 patients in the ANCHOR trial experienced 2+ to 4+ inflammation on slit-lamp examination and were not treated for presumed endophthalmitis. In contrast, at our institution, nearly all patients who develop vitritis, or who develop significant anterior chamber cellular reaction, would be given intravitreal antibiotics. Including these untreated patients, the clinically presumed endophthalmitis rate increases to $0.18 \%$ in the MARINA trial and $0.22 \%$ in the ANCHOR trial. It is possible that our study includes eyes with postinjection inflammation that would have been observed in the MARINA and ANCHOR trials.

Endophthalmitis rates in retrospective studies vary tremendously. Fintak and colleagues $^{14}$ identified cases of endophthalmitis from billing records at four institutions, reporting a rate of $0.02 \%$ ( 6 of 26,905 injections). All injecting physicians used a lid speculum and $5 \%$ to $10 \%$ topical povidone-iodine drops to disinfect the ocular surface; some physicians used $10 \%$ povidone-iodine soaked swabs to clean the eyelid skin, eyelashes, and lid margin. Pilli and colleagues ${ }^{8}$ also reported a similarly low rate of postinjection endophthalmitis in an office setting $(0.029 \%, 3$ of 10,254 injections). In this study, the authors retrospectively collected endophthalmitis cases by reviewing case notes and from conversations with referral sources and other vitreoretinal groups in the area. Patients were prepped with 5\% povidone-iodine drops. A lid speculum was used based on the surgeon's discretion. In both of these studies, the retrospective study design could have missed endophthalmitis cases, underestimating the incidence of this rare 
complication. At the other end of the spectrum, Fong and colleagues ${ }^{15}$ reported a 10 -fold 2 higher rate of endophthalmitis in a retrospective study of intravitreal bevacizumab and 3 ranbizumab injections ( $0.26 \%, 4$ of 1,553 total injections), collecting cases from an AMD 4 registry amassed from injection logs. Details were not given regarding the injection 5 technique.

Non-infectious endophthalmitis, or uveitis, has been reported after intravitreal anti-VEGF injection, particularly after bevacizumab injection ${ }^{9}, 10,16,17$. In our study, however, the proportion of culture-negative - and possibly non-infectiousendophthalmitis cases was similar after bevacizumab and ranibizumab injections.

Prior studies have offered clinical criteria to distinguish between culture-positive and culture-negative endophthalmitis. Ness and colleagues ${ }^{9}$ reported 10 cases of uveitis, termed toxic vitritis, after bevacizumab injection. They felt the timing and severity of pain helped distinguish it from infectious endophthalmitis. All toxic vitritis cases presented within 48 hours with mild to no pain. A hypopyon was not a distinguishing feature; six cases of toxic vitritis presented with hypopyon. The authors attributed these cases to a toxic reaction from the brand of syringe used for injection. Georgopoulos and colleagues ${ }^{10}$ reported eight cases of non-infectious endophthalmitis after bevacizumab. All cases presented within two days of injection without hypopyon. Only one patient had pain. Mezad-Koursh and colleagues found that later presentation, pain, keratic precipitates, fibrin, hypopyon, and anterior synechiae were more typical of culture positive endophthalmitis ${ }^{18}$.

In contrast, our study suggests that one cannot clinically distinguish between culture-positive and culture-negative endophthalmitis after anti-VEGF injection. All cases in our series had pain, decreased vision, and vitritis. Both culture-positive and culture-negative cases presented an average of three to four days after injection. Most patients in both groups had a hypopyon. Anecdotally, one case of endophthalmitis due to Streptococcus viridans with a final visual acuity of no light perception initially presented two days after injection with $3+$ cell and no hypopyon. Another patient presented with sequential hypopyon endophthalmitis after bevacizumab. The first episode resolved to baseline visual acuity six weeks after tap and inject. The second episode did not improve with hourly topical prednisolone acetate, and required tap and inject to control the inflammation; the vision never returned to baseline visual acuity at six months. We suggest that presumed infectious endophthalmitis should be considered in all instances with post-injection inflammation in the vitreous cavity greater than 1+ cell, and strong consideration should be given to treating these cases with emergent tap and injection of intravitreal antibiotics.

Although most cases with endophthalmitis after intravitreal anti-VEGF injection returned to baseline visual acuity within three to six months, $17 \%$ lost more than two lines at final follow-up. These outcomes are similar to those reported by Klein and colleagues $^{6}$, and worse than those in other smaller studies ${ }^{8,19}$. There was no significant difference in rates of visual recovery between culture-positive and culture-negative cases. Only a small percentage of cases (17\%) required pars plana vitrectomy.

Several authors have emphasized the role of specific aspects of prepping technique to prevent endophthalmitis after intravitreal injection. The only proven endophthalmitis prophylaxis remains topical povidone-iodine to sterilize the ocular surface $^{20,21}$. It is important to sterilize the ocular surface with povidone-iodine before 
applying a viscous anesthetic; viscous gel can form a barrier preventing povidone-iodine from coming in contact with conjunctival bacteria ${ }^{22,23}$. Further, physicians and patients should avoid talking, coughing, and sneezing during anti-VEGF injection administration to prevent contamination with oral flora ${ }^{24,25}$. Streptococcus species isolates, bacteria commonly found in oral flora and isolated in two of our cases, occur three to four times more frequent in endophthalmitis after intravitreal injection than after intraocular surgery ${ }^{24,25}$.

The VEGF Inhibition Study in Ocular Neovascularization (VISION) trial ${ }^{26}$ investigators felt the risk of post-injection endophthalmitis could be modified by vigilance to an aseptic injection technique. Their initial endophthalmitis rate was $0.18 \%$ per injection (13 cases in 7,171 injections). After amending the injection protocol to include a sterile drape and an additional pre-injection antibiotic or povidone-iodine flush, rates decreased to $0.04 \%(2$ of 4,465$)$ at centers adopting the amended protocol. They attributed $75 \%$ of cases ( 9 of 12) to the failure of using a lid speculum. Many authors recommend use of a bladed lid speculum ${ }^{27-30}$, though this recommendation is based on the theoretical benefit of covering the eyelashes and eyelids from touching the needles and injection site, and not on empiric evidence. Others argue that insertion of a lid speculum can massage secretions from meibomian glands, thus contaminating the ocular surface $^{30}$. Mason and colleagues ${ }^{31}$ recently reported in a prospective masked randomized trial of 174 patients undergoing intravitreal injection that lid speculum use did not result in an increase in conjunctival bacterial counts (paired t-test, $\mathrm{p}=0.9455$ ). Our study found no difference in endophthalmitis rates when comparing injections administered with and without a bladed lid speculum. All of the studies to date, including ours with a relatively large sample size, are underpowered to detect smaller differences in the rate of endophthalmitis due to the low incidence of endophthalmitis. Over 100,000 injections would need to be administered in order to find a difference in endophthalmitis rate of $0.05 \%$ and $0.10 \%$.

There is some debate as to the whether hemisphere or quadrant of injection affects endophthalmitis rates. Superior hemisphere injections tend to be covered by the upper eyelid, away from a potentially contaminated lid margin and meibomian glands.

Additionally, this location allows masking of incidental subconjunctival hemorrhage by the upper eyelid. The disadvantage of superior hemisphere injections is the difficulty of administering the injection when patients attempt to squeeze their eyes with resultant Bell's reflex and supraduction. Those who inject in the inferior hemisphere often find good exposure. Further, the upward gaze required by inferior hemisphere injection thins the inferior tear film, theoretically decreasing the concentration of bacteria ${ }^{8}$. On the other hand, other ocular surgeries, such as inferiorly placed trabeculectomies, carry an increased risk of endophthalmitis compared to those placed superiorly ${ }^{32,33}$, a finding attributed to the bacteria-rich tear film ${ }^{34}$. Roth and colleagues ${ }^{35}$ reported a greater risk of endophthalmitis after inferior hemisphere injection compared to those in the superior hemisphere among 10,834 consecutive injections. Our study found no difference in endophthalmitis risk between superior and inferior hemisphere injections, suggesting either hemisphere is acceptable.

Some vitreoretinal specialists displace the conjunctiva with a sterile cotton tip applicator when injecting through the pars plana in an effort to avoid a straight tract for bacteria to enter through the conjunctiva and sclera into the vitreous cavity ${ }^{36}$. Others 
argue it is best to minimize manipulation of the ocular surface to decrease risk of potential contamination. In our study, there was no difference in endophthalmitis risk between those who do and do not displace conjunctiva while injecting.

There was no difference in endophthalmitis risk after bevacizumab or ranibizumab injection in our study, similar to the findings of other studies ${ }^{6,8}$. Given the wide confidence intervals, however, we cannot draw strong conclusions from this result.

Our study has several limitations. Although we identified and recorded endophthalmitis cases prospectively with an infection surveillance program, a method we feel is more accurate than retrospective identification, it is possible that we underestimated risk of endophthalmitis. We retrospectively reviewed charts at the end of the surveillance period, which could have introduced certain biases and inaccuracies. For example, our study utilized Snellen acuity, which is not as accurate as best-corrected visual acuity. Also, we were unable to assess other relevant risk factors, such as degree of blepharitis, because this was not systematically documented in the charts. Our culturepositivity rate of $30.4 \%$ was lower compared to other studies. For example, the Endophthalmitis Vitrectomy Study (EVS) ${ }^{37}$ reported that $66 \%$ of cases (138 of 202) undergoing tap and inject for endophthalmitis after cataract surgery were confirmed culture-positive. Their higher rate of culture-positivity may be related to their methodology; they collected vitreous samples by either single port vitrectomy or needle aspiration whereas we only used needle apiration. In our study, nine of 23 cases had an unsuccessful vitreous biopsy and thus had aqueous biopsy alone, and in the EVS, aqueous biopsy was associated with a lower confirmed laboratory infection rate (26.9\%) compared to undiluted vitreous $(58.9 \%)^{38}$.

Another possible reason our culture-positivity rate was low could be that we included cases of presumed non-infectious endophthalmitis. Intraocular inflammation is a known possible sequeale of intravitreal anti-VEGF injection ${ }^{10,39}$. Our standard practice is to administer intravitreal antibiotics whenever the examing physician feels that the case is more likely then non-infectious endophthalmitis.

Because of the low incidence of endophthalmitis, our risk factor analysis is underpowered to find small differences. It is possible that our risk factor results are subject to misclassification bias if the injecting vitreoretinal specialists deviated from their preferred injection technique during some injections. Further, there may have been undocumented variations in prepping technique in cases developing endophthalmitis.

In summary, the risk of endophthalmitis after intravitreal anti-VEGF injection is low. The accuracy of reported rates in the literature, in part, depends on individual study designs and the study's definition of "endophthalmitis". Visual outcomes are good for most cases, with $83 \%$ to baseline visual acuity within three to six months. However, a subgroup of infected eyes will have devastating visual outcomes. The presence or absence of pain, vitritis, decreased vision, or hypopyon, and the interval between injection and presentation, does not help distinguish culture-positive from culturenegative cases. Thus, we recommend vitreoretinal specialists have a low threshold to perform emergent tap and injection of intravitreal antibiotics. This study did not identify any modifiable risk factors to prevent endophthalmitis. The incidence endophthalmitis does not appear to be affected by use of a lid speculum, conjunctival displacement, hemisphere of injection, or use bevacizumab or ranbizumab. 


\section{$\underline{\text { References }}$}

1. Brown DM, Campochiaro PA, Singh RP, et al. Ranibizumab for macular edema following central retinal vein occlusion: six-month primary end point results of a phase III study. Ophthalmology 2010;117:1124-33.

2. Campochiaro PA, Heier JS, Feiner L, et al. Ranibizumab for macular edema following branch retinal vein occlusion: six-month primary end point results of a phase III study. Ophthalmology 2010;117:1102-12.

3. Gheith ME, Siam GA, de Barros DS, et al. Role of intravitreal bevacizumab in neovascular glaucoma. J Ocul Pharmacol Ther 2007;23:487-91.

4. Elman MJ, Aiello LP, Beck RW, et al. Randomized trial evaluating ranibizumab plus prompt or deferred laser or triamcinolone plus prompt laser for diabetic macular edema. Ophthalmology 2010;117:1064-77 e35.

5. Pharmakakis NM, Petropoulos IK, Georgakopoulos CD, et al. Apoptotic mechanisms within the retina in Staphylococcus epidermidis experimental endophthalmitis. Graefes Arch Clin Exp Ophthalmol 2009;247:667-74.

6. Klein KS, Walsh MK, Hassan TS, et al. Endophthalmitis after anti-VEGF injections. Ophthalmology 2009;116:1225.

7. Diago T, McCannel CA, Bakri SJ, et al. Infectious endophthalmitis after intravitreal injection of antiangiogenic agents. Retina 2009;29:601-5.

8. Pilli S, Kotsolis A, Spaide RF, et al. Endophthalmitis associated with intravitreal anti-vascular endothelial growth factor therapy injections in an office setting. Am J Ophthalmol 2008;145:879-82.

9. Ness T, Feltgen N, Agostini H, et al. Toxic vitreitis outbreak after intravitreal injection. Retina;30:332-8.

10. Georgopoulos M, Polak K, Prager F, et al. Characteristics of severe intraocular inflammation following intravitreal injection of bevacizumab (Avastin). Br J Ophthalmol 2009;93:457-62.

11. Rosenfeld PJ, Brown DM, Heier JS, et al. Ranibizumab for neovascular agerelated macular degeneration. N Engl J Med 2006;355:1419-31.

12. Brown DM, Michels M, Kaiser PK, et al. Ranibizumab versus verteporfin photodynamic therapy for neovascular age-related macular degeneration: Two-year results of the ANCHOR study. Ophthalmology 2009;116:57-65 e5.

13. Brown DM, Kaiser PK, Michels M, et al. Ranibizumab versus verteporfin for neovascular age-related macular degeneration. N Engl J Med 2006;355:1432-44.

14. Fintak DR, Shah GK, Blinder KJ, et al. Incidence of endophthalmitis related to intravitreal injection of bevacizumab and ranibizumab. Retina 2008;28:1395-9.

15. Fong DS, Custis P, Howes J, Hsu JW. Intravitreal bevacizumab and ranibizumab for age-related macular degeneration a multicenter, retrospective study. Ophthalmology 2010;117:298-302.

16. Yamashiro K, Tsujikawa A, Miyamoto K, et al. Sterile endophthalmitis after intravitreal injection of bevacizumab obtained from a single batch. Retina 2010;30:48590.

17. Wickremasinghe SS, Michalova K, Gilhotra J, et al. Acute intraocular inflammation after intravitreous injections of bevacizumab for treatment of neovascular age-related macular degeneration. Ophthalmology 2008;115:1911-5. 
18. Mezad-Koursh D, Goldstein M, Heilwail G, et al. Clinical characteristics of endophthalmitis after an injection of intravitreal antivascular endothelial growth factor. Retina 2010;30:1051-7. 19. Mason JO, 3rd, White MF, Feist RM, et al. Incidence of acute onset endophthalmitis following intravitreal bevacizumab (Avastin) injection. Retina 2008;28:564-7.

20. Speaker MG, Menikoff JA. Prophylaxis of endophthalmitis with topical povidone-iodine. Ophthalmology 1991;98:1769-75.

21. Moss JM, Sanislo SR, Ta CN. A prospective randomized evaluation of topical gatifloxacin on conjunctival flora in patients undergoing intravitreal injections. Ophthalmology 2009;116:1498-501.

22. Boden JH, Myers ML, Lee T, et al. Effect of lidocaine gel on povidone-iodine antisepsis and microbial survival. J Cataract Refract Surg 2008;34:1773-5.

23. Awotesu S, Eke T. Preoperative lidocaine gel. Ophthalmology;117:1049.

24. McCannel CA. Meta-analysis of endophthalmitis after intravitreal injection of anti-VEGF agents: causative organisms and possible prevention strategies. Presented at American Society of Retina Specialists, September 1, 2010, Vancouver, Canada.

25. Chen E LM, Cox J, Brown DM. Ten years of endophthalmitis at a single tertiary retina practice: comparing intravitreal injection cases vs. post-anterior segment surgery cases. Presented at American Society of Retina Specialists, September 1, 2010, Vancouver, Canada.

26. D'Amico DJ, Masonson HN, Patel M, et al. Pegaptanib sodium for neovascular age-related macular degeneration: two-year safety results of the two prospective, multicenter, controlled clinical trials. Ophthalmology 2006;113:992-1001. 27. Ta CN. Minimizing the risk of endophthalmitis following intravitreous injections. Retina 2004;24:699-705.

28. Scott IU, Flynn HW, Jr. Reducing the risk of endophthalmitis following intravitreal injections. Retina 2007;27:10-2.

29. Bhavsar AR, Googe JM, Jr., Stockdale CR, et al. Risk of endophthalmitis after intravitreal drug injection when topical antibiotics are not required: the diabetic retinopathy clinical research network laser-ranibizumab-triamcinolone clinical trials. Arch Ophthalmol 2009;127:1581-3.

30. Aiello LP, Brucker AJ, Chang S, et al. Evolving guidelines for intravitreous injections. Retina 2004;24:S3-19.

31. Mason JO, 3rd, Friedman DA, Finley TA, et al. Timing of Povidone-Iodine Prophylaxis for Intravitreal Injections. Presented at the American Academy of Ophthalmology Annual Meeting, October 18, 2010, Chicago, Illinois.

32. Higginbotham EJ, Stevens RK, Musch DC, et al. Bleb-related endophthalmitis after trabeculectomy with mitomycin C. Ophthalmology 1996;103:650-6.

33. Mac I, Soltau JB. Glaucoma-filtering bleb infections. Curr Opin Ophthalmol 2003;14:91-4.

34. Dunnington JH, Locatcher-Khorazo D. Value of cultures before operation for cataract. Arch Ophthalmol 1945;34:215-9.

35. Roth D, Gowtham J, Kheterpal A, et al. Inferior Intravitreal Injection Site Associated With Higher Incidence of Post-injection Endophthalmitis. Presented at 
American Academy of Ophthalmology Annual Meeting, October 25, 2009, San

Francisco, CA.

36. Garg SJ, Recchia FM. Re: Evolving guidelines for intravitreous injections. Retina 2005;25:949-50.

37. Results of the Endophthalmitis Vitrectomy Study. A randomized trial of immediate vitrectomy and of intravenous antibiotics for the treatment of postoperative bacterial endophthalmitis. Endophthalmitis Vitrectomy Study Group. Arch Ophthalmol 1995;113:1479-96.

38. Barza M, Pavan PR, Doft BH, et al. Evaluation of microbiological diagnostic techniques in postoperative endophthalmitis in the Endophthalmitis Vitrectomy Study. Arch Ophthalmol 1997;115:1142-50.

39. Bakri SJ, Larson TA, Edwards AO. Intraocular inflammation following intravitreal injection of bevacizumab. Graefes Arch Clin Exp Ophthalmol 2008;246:77981. 